Supporting Information 


\section{A comparison of metal adhesion layers for Au films in thermo-plasmonic applications}

William M. Abbott ${ }^{1}$, Christopher P. Murray ${ }^{1}$, Sorcha Ní Lochlainn ${ }^{1}$, Frank Bello ${ }^{1}$, Chuan Zhong ${ }^{1}$, Christopher Smith ${ }^{1}$, Eoin K. McCarthy ${ }^{2}$, Clive Downing ${ }^{2}$, Dermot Daly ${ }^{2}$, Amanda K. Petford-Long ${ }^{3,4}$, Cormac McGuinness ${ }^{1}$, Igor Igorovich Chunin ${ }^{1}$, John F. Donegan ${ }^{1}$, David McCloskey ${ }^{*}$

${ }^{1}$ School of Physics, CRANN and AMBER, Trinity College Dublin, Dublin 2, Ireland.

${ }^{2}$ Advanced Microscopy Laboratory \& AMBER, Trinity College Dublin, Dublin 2, Ireland.

${ }^{3}$ Material Science Division. Argonne National Laboratory, Lemont, IL 60439, USA

${ }^{4}$ Department of Materials Science and Engineering, Northwestern University, Evanston, IL 60208, USA

\section{SUPPORTING DATA}

Atomic force microscopy (AFM) was used to characterize the surface topography of the annealed samples of Ta, $\mathrm{Ti}$ and W/Au (50nm), and the results are shown in Figure S1. The route mean square roughness values (Ra) for the surface of the samples with $0.5 \mathrm{~nm}$ adhesion layer are quite similar. The samples with $5 \mathrm{~nm}$ adhesion layers tend to be rougher, with Ti exhibiting the highest Ra value and $\mathrm{W}$ the lowest. This relates to the extent of the diffusion of adhesion metals through the Au films upon annealing, and correlates with other observations (TEM, XPS). 
(a) $\mathrm{Ra}=0.49 \mathrm{~nm}$

$\mathrm{Ta}$

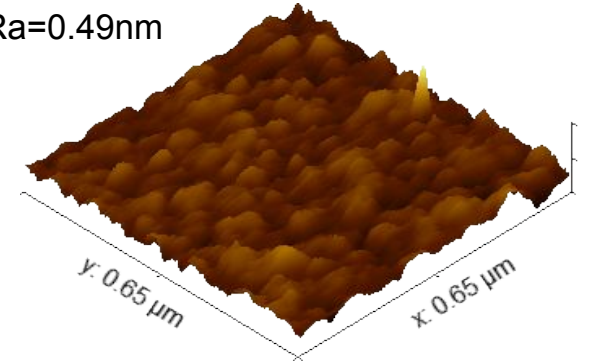

(c) $R a=0.53 \mathrm{~nm}$

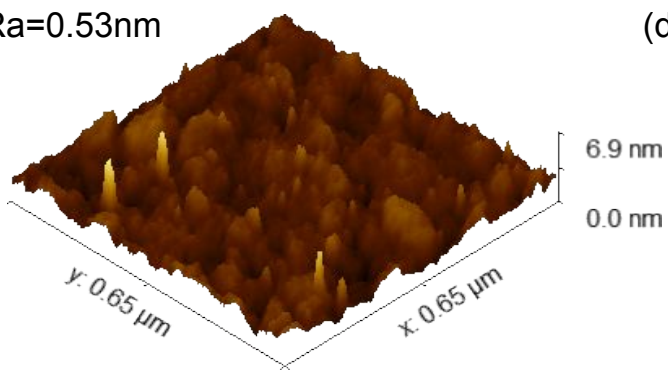

(e) $\mathrm{Ra}=0.60 \mathrm{~nm}$

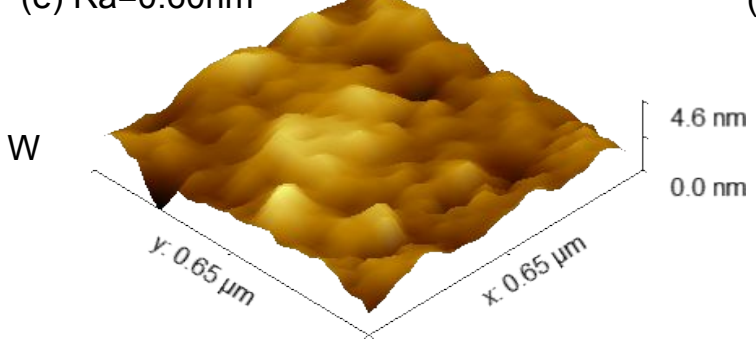

$0.5 \mathrm{~nm}$

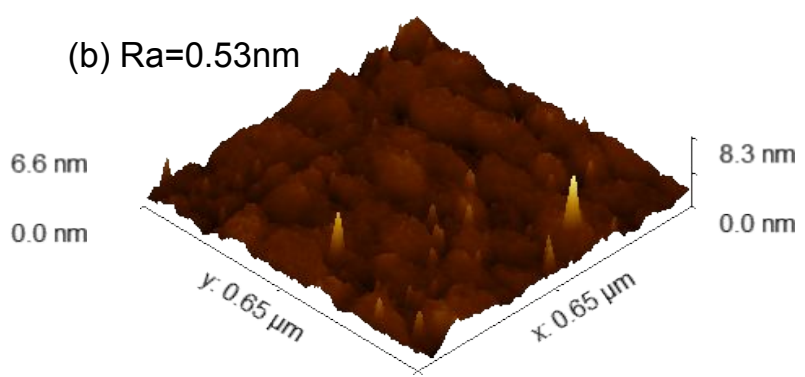

(d) $\mathrm{Ra}=2.12 \mathrm{~nm}$

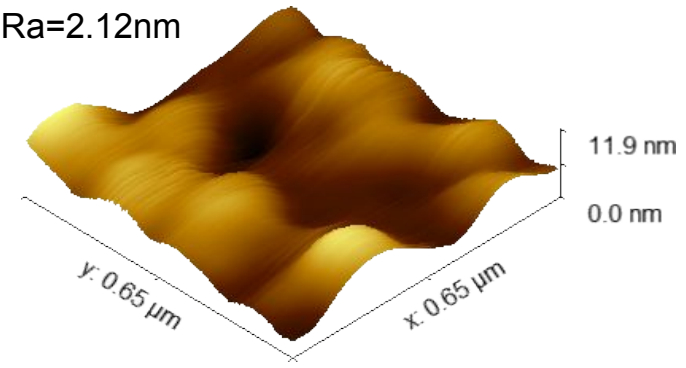

(f) $\mathrm{Ra}=0.48 \mathrm{~nm}$

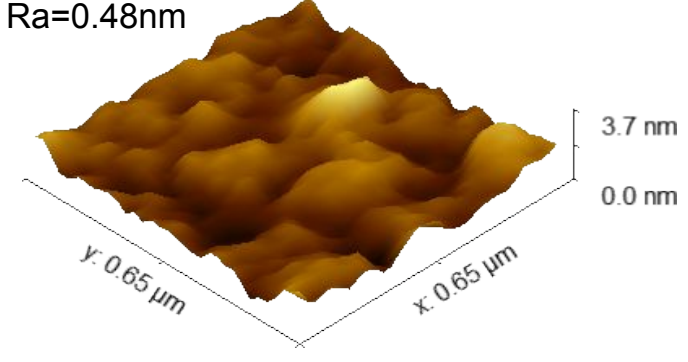

$5 \mathrm{~nm}$

Figure S1AFM topographical images of samples annealed in air for 10 hours at 250C. (a) Ta 5A Au 50nm, (b) Ta 5nm Au 50nm, (c) Ti 5A Au 50nm, (d) Ti 5nm Au 50nm, (e) W 5A Au 50nm, (f) W 5nm Au 50nm. RMS roughness (Ra) values are inset.

EDX data for Ti/Au (Figure S2) and W/Au () samples were measured on electron transparent TEM lamellae. All samples were annealed at $250^{\circ} \mathrm{C}$ for 10 hours in air. EDX was not carried out on the Ta/Au system due to overlap in peak positions between $\mathrm{Ta}$ and $\mathrm{Au}$.

For the $\mathrm{Ti}(0.5 \mathrm{~nm}) / \mathrm{Au}(50 \mathrm{~nm})$ sample, the Ti layer is detectable over a narrow $(\approx 2 \mathrm{~nm})$ transition, but not in the Au over-layer (Figure S2 (a) and (b)). In contrast, Figure S2 (c), (d) and (e) show that the Ti (5nm) layer has clearly diffused completely though the Au grain boundaries to the Au/air interface, where it readily oxidises and undergoes volume expansion leading to compressive stress in the Au film. The integrity of the Au layer has clearly been compromised and the film begins to dewet.

In contrast, there is no EDX evidence of W diffusion through the Au layer for either W (0.5nm)/ Au (50nm) or $\mathrm{W}(5 \mathrm{~nm}) / \mathrm{Au}(50 \mathrm{~nm})$ (Figure S3). 

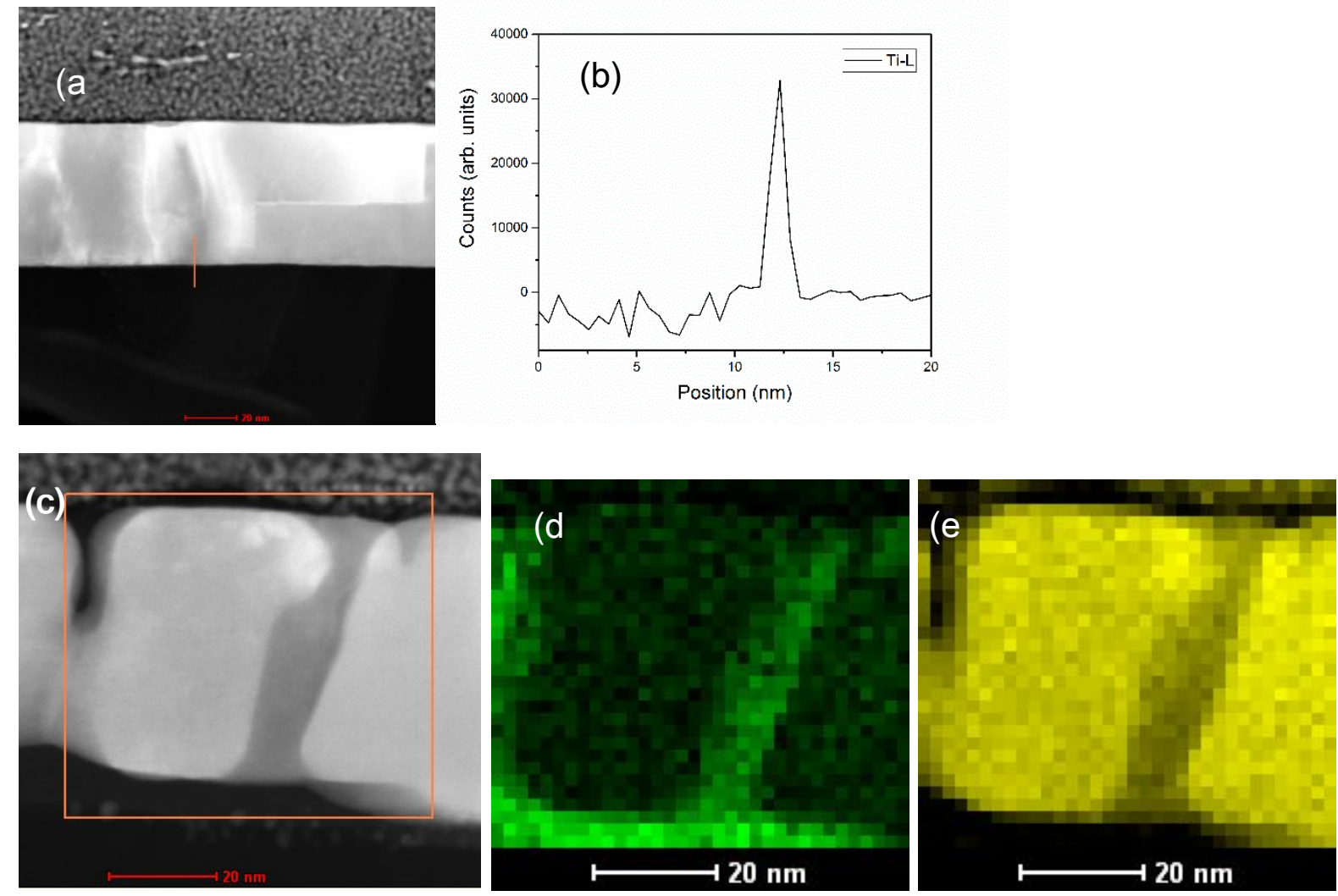

Figure S2. STEM/EDX/EELS analysis of Ti/Au TEM lamellae. (a) STEM image Ti (0.5nm)/ Au (50nm) post anneal 10 hours at 250C with EELS line scan marked in red. (b) EELS line scan data for Ti from image (a); (c) STEM image Ti (5nm) / Au (50nm) post anneal 10 hours at 250C with EDX area map marked in orange; (d) EDX data for Ti from image (c); (e) EDX data for Au from image (c)
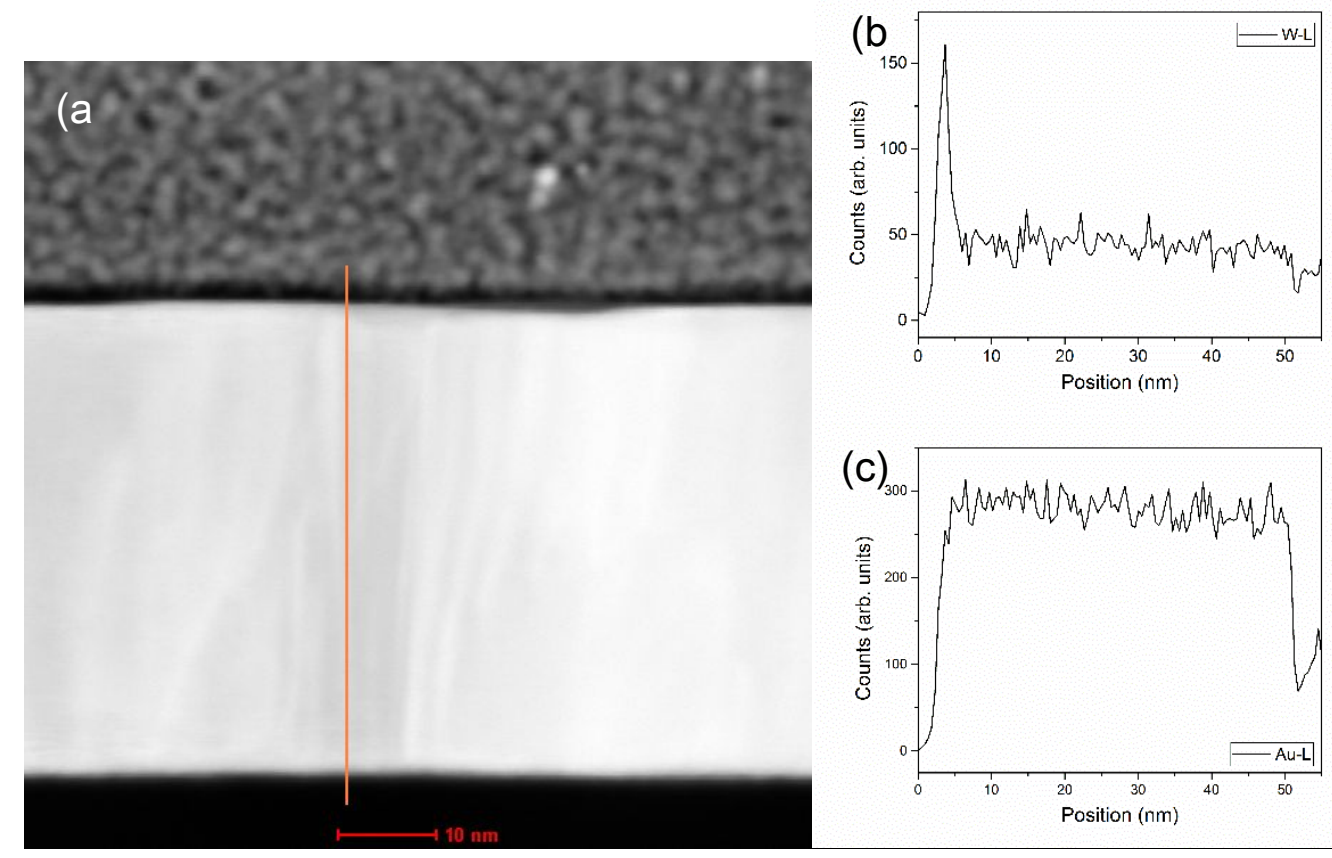

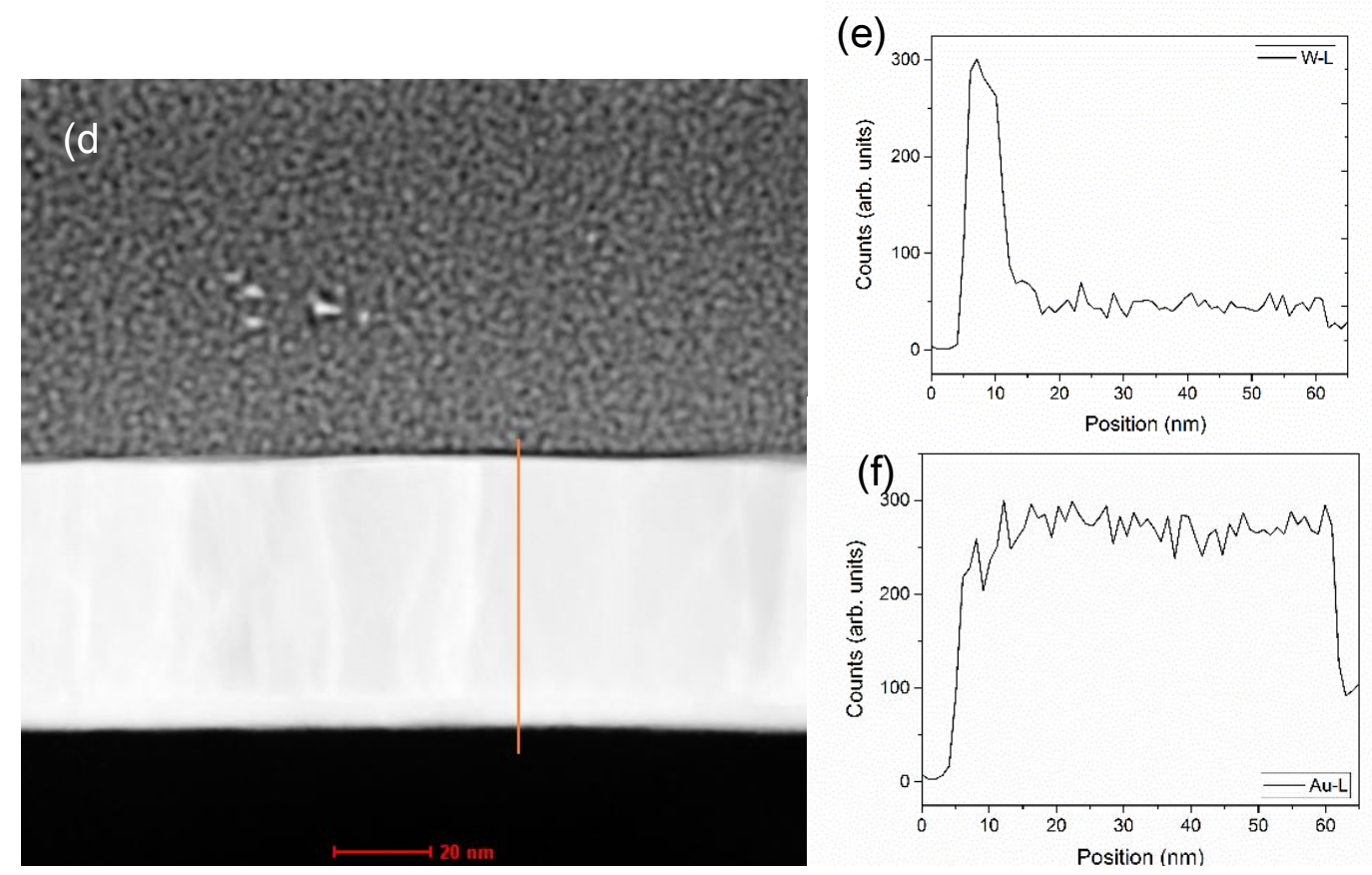

Figure S3. STEMIEDX images for the WIAu system post anneal at 250C for 10 hours in air; (a) STEM cross section of the $0.5 \mathrm{~nm}$ W/ 50nm Au sample; (b) EDX data for W for linescan shown in (a). The increased background is an artefact of the high density of the Au layer; (c) EDX data for Au for linescan shown in (a); (d) STEM cross section of $5 \mathrm{~nm}$ W/ $50 \mathrm{~nm}$ Au sample; (e)EDX data for W for linescan shown in (d) ; (f) EDX data for Au for linescan shown in (d).

XPS data for $\mathrm{Ta} / \mathrm{Au}$ and W/Au samples and angle-resolved XPS (ARXPS) for Ta/Au samples were measured on an Omicron EA125 electron spectrometer system equipped with a monochromated Al K $\alpha$ source (XM-1000) and processed with CasaXPS. The samples were introduced from ex-situ post-annealing with no in-situ surface modification. The ARXPS was obtained from take-off angles of $90^{\circ}$ to $30^{\circ}$ and at high transmission pass energies of $100 \mathrm{eV}$ for Au 4p, O 1s, Au 4d, C 1s, Au 4f and Ta 4f core levels to ensure good signal to noise ratios and good statistics. The electron analyzer's angular acceptance was set to $\pm 1^{\circ}$ for these ARXPS measurements

Results obtained from the ARXPS data of the $5 \mathrm{~nm} \mathrm{Ta} / 50 \mathrm{~nm}$ Au sample are shown in Figure S4(a) where the relative-sensitivity factor weighted area intensity rations of $\mathrm{Au} 4 \mathrm{p}: \mathrm{O}$ 1s; of $\mathrm{Au} 4 \mathrm{~d}: \mathrm{C} 1 \mathrm{~s}$ and of $\mathrm{Au} 4 \mathrm{f}: \mathrm{Ta} 4 \mathrm{f}$ clearly increase at similar rates on going to more surface-sensitive lower TOA. Excluding the $\mathrm{C} 1 \mathrm{~s}$ and $\mathrm{O} 1 \mathrm{~s}$ contributions the weighted fractional area intensity of $\mathrm{Ta} 4 \mathrm{f}$ as compared to $\mathrm{Au} 4 \mathrm{f}$ is seen to increase from $\sim 2.5 \%$ to $\sim 6.5 \%$ of the combined $\mathrm{Au}$ and $\mathrm{Ta} 4 \mathrm{f}$ intensities for the lower TOA as is shown in Figure S4(b).
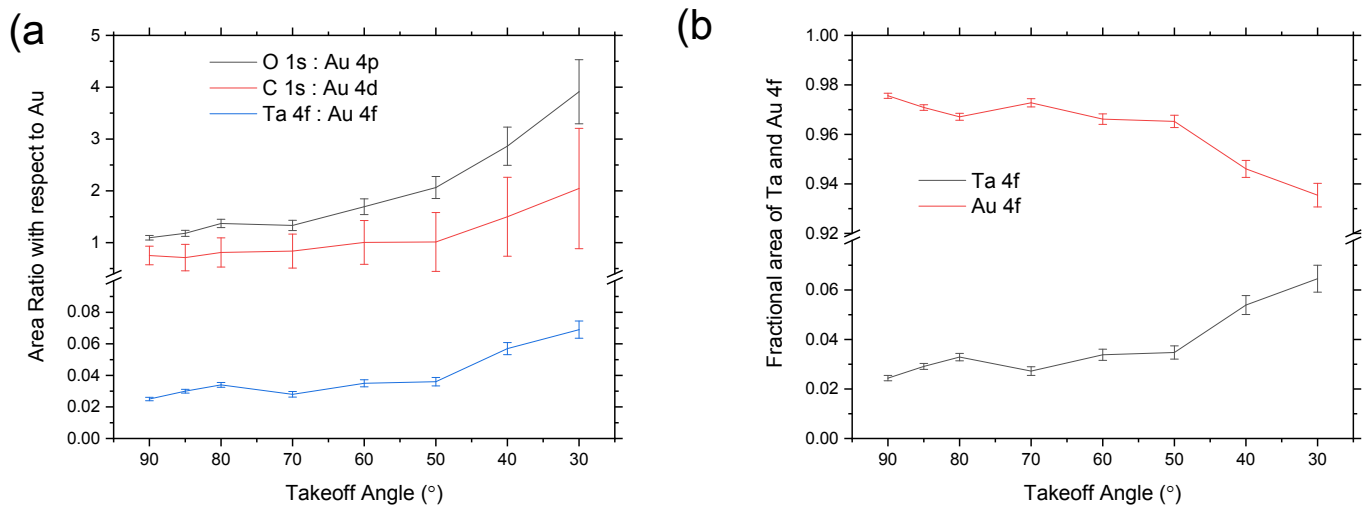
Figure S4. ARXPS data for the $5 \mathrm{~nm}$ Ta $150 \mathrm{~nm}$ Au sample post anneal at 250C for 10 hours in air; (a) The weighted area ratios of the $O$ 1s: Au 4p3/2 (black), of the C 1s : Au 4d5/2 peaks (red) and of the Au 4f: Ta 4f regions (blue) and their variation with decreasing take-off angle as the ARXPS becomes more surface sensitive. (b) The percentage of combined weighted area between the Au $4 f$ and Ta $4 f$ as the take-off angle decreases.

From our knowledge of the distribution and source of the tantalum diffusing upwards from the adhesion layer as indicated in the STEM/EDX images it is not appropriate to attempt to fit the ARXPS data to a simple overlayer model. Nevertheless, an upper limit on the thickness of the surface layer of $\mathrm{Ta}_{2} \mathrm{O}_{5}$ can be estimated using for instance the thickogram method of Cumpson ${ }^{1}$, giving a maximal thickness (if a single layer and species) of 0.07 nm or perhaps 0.15 of a monolayer.

Figure S5 shows the complete set of ARXPS spectra obtained from the $5 \mathrm{~nm}$ Ta / $50 \mathrm{~nm}$ Au annealed sample and how the four spectral regions acquired at each angle have changing intensities of the chemical species present with differing TOA. This data is summarized in Figure S3. In addition, it can be seen that the Ta $4 \mathrm{f}$ region changes in spectral shape with differing TOA. In normal photoemission with the TOA being equal to $90^{\circ}$, the sampling depth for a uniformly solid film with no open areas would be of the order of three times the inelastic mean free path, which in the case of the Ta 4 f region would be $7.2 \mathrm{~nm}^{2,3,4}$. At a TOA of $30^{\circ}$, the nominal sampling depth is half this amount. In the case of a film with a small proportion of open area and with grain boundary fissures, a larger proportion of Ta derived photoemission may arise from these inner surfaces than would otherwise be the case for normal and near-normal photoemission geometries which might allow photoelectrons to escape from greater depths but without being strongly attenuated as expected from those depths in a solid system. The predominant tantalum oxide seen at these near-normal photoemission TOAs (near $90^{\circ}$ ) is $\mathrm{Ta}_{2} \mathrm{O}_{5}$ as observed in the high resolution XPS spectrum shown in Figure 5(b) where it accounts for $81 \%$ of the observed spectral weight due to tantalum species. The variation of the tantalum oxide components with TOA is seen in the fourth panel of Figure S5. In particular, the contrast between the extremal end-points of the ARXPS measurements is illustrated in the last panel of Figure S5 where the binding energies even for the lower resolution $100 \mathrm{eV}$ pass energy ARXPS spectra clearly indicate that the predominant contribution from the surface has changed. At the lowest TOAs, the predominant tantalum oxide contribution to the $30^{\circ}$ spectrum has become $\sim 72 \% \mathrm{Ta}_{2} \mathrm{O}_{3}$ rather than $\sim 70 \% \mathrm{Ta}_{2} \mathrm{O}_{5}$ in the $90^{\circ} \mathrm{TOA}$ spectrum. Note that the differing estimates for the $\mathrm{Ta}_{2} \mathrm{O}_{5}$ contribution at $90^{\circ}$ is due to the very different spectral resolutions between the ARXPS spectra of $3.0 \mathrm{eV}$ and the high resolution spectrum of Figure 5 (b) of $0.7 \mathrm{eV}$. Nevertheless, the ARXPS data indicates that the tantalum oxide on the surface is less fully oxidized than that observed from deeper into the bulk of the $5 \mathrm{~nm} \mathrm{Ta} / 50 \mathrm{~nm}$ Au film from the film/air interface. 

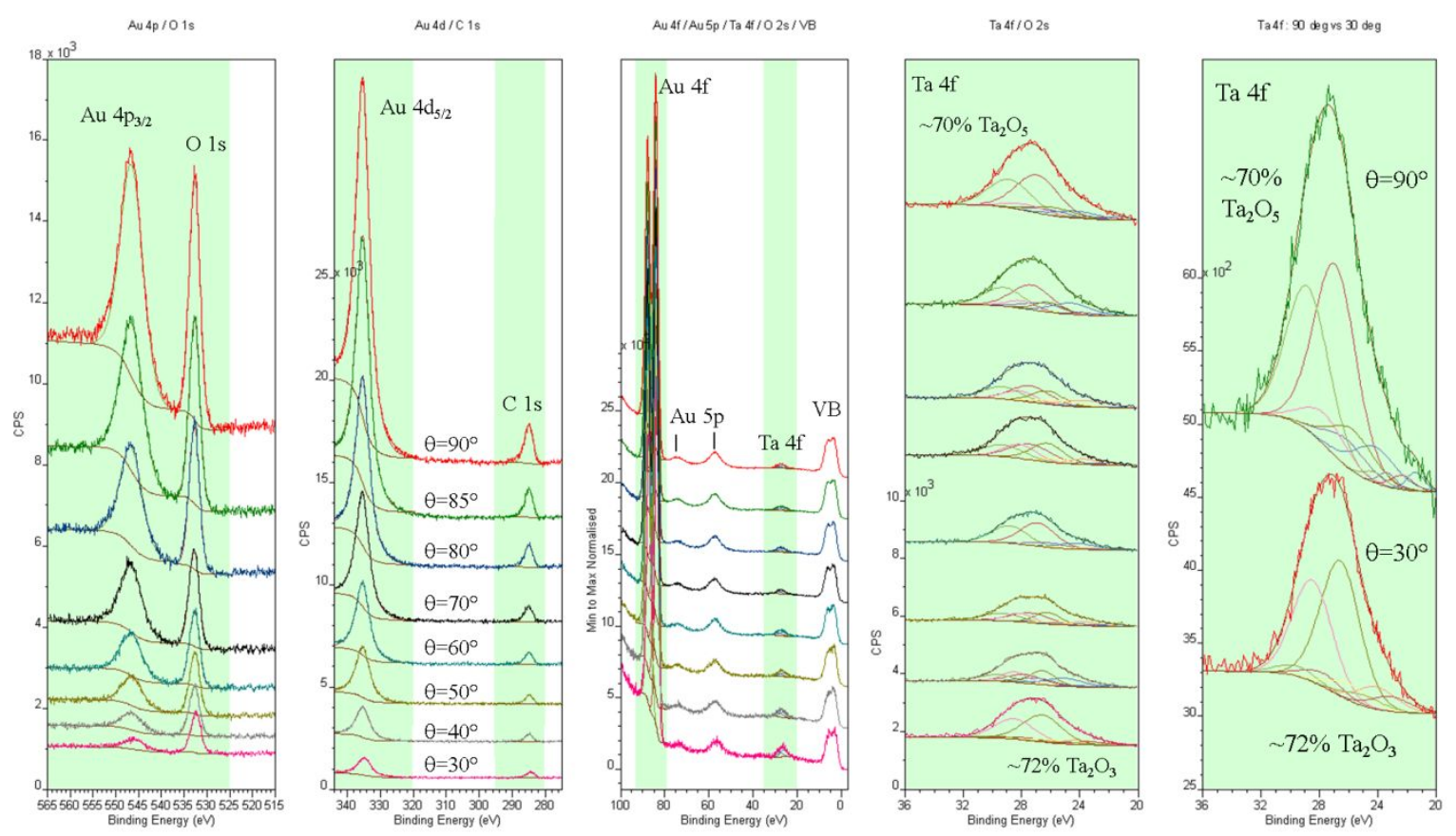

Figure S5. ARXPS data for the $5 \mathrm{~nm}$ Ta $/ 50 \mathrm{~nm}$ Au sample post anneal at 250C for 10 hours in air; From left to right: (a) The Au 4p $p_{3 / 2}$ and $O$ 1s photoelectron peaks at differing TOA offset for clarity; (b) The Au $4 d_{5 / 2}$ and $C 1 s$ peaks at differing TOA; (c) the Au 4f, Au 5p, Ta $4 f$ and valence band (VB) regions at differing TOA; (d) the Ta $4 f$ region obtained with differing TOA. The positions of the spin-orbit split Ta 4 f components are as previously indicated in Figure 5(b).; (e) The extremal end points for the ARXPS of the Ta 4 region illustrating the shift from $\mathrm{Ta}_{2} \mathrm{O}_{5}$ at $90^{\circ}$ to $\mathrm{Ta}_{2} \mathrm{O}_{3}$ at $30^{\circ}$ as the take-off angle decreases and the ARXSP is moste surface sensitive. 


\section{REFERENCES}

${ }^{1}$ P.J. Cumpson, Surf. Interface Anal. 29, 403 (2000).

${ }^{2}$ C.J. Powell and A. Jablonski, NIST Electron Inelastic-Mean-Free-Path Database: Version 1.2 (National Institute of Standards and Technology, Gaithersburg, Maryland, 2010).

${ }^{3}$ C.J. Powell and A. Jablonski, J. Phys. Chem. Ref. Data 28, 19 (1999).

${ }^{4}$ S. Tanuma, C.J. Powell, and D.R. Penn, Surf. Interface Anal. 17, 911 (1991). 